\title{
HISTORIA
}

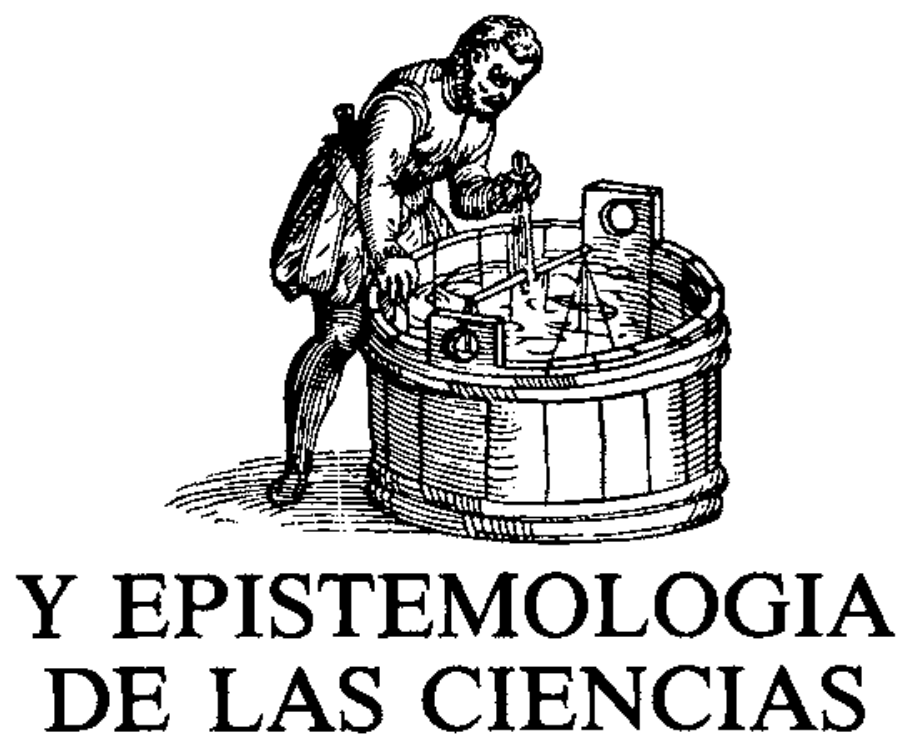

\section{EL CONCEPTO DE MASA EN LA FÍSICA CLÁSICA: ASPECTOS HISTÓRICOS Y DIDÁCTICOS}

DOMÉNECH, A.

I.B. Buñol. València. Av̛da. Rafel Ridaura, 1. 46360 Buñol. València.

\section{SUMMARY}

Different definitions of mass in classical physics are discussed in basis to their physical representation. The following questions are adressed: $a$ ) the quantitas materiae protlem, $b$ ) dichotomy between inertial and gravitational masses and, c) didactic implications of the different definitional modalities.

\section{INTRODUCCIÓN}

La reciente investigación didáctica ha puesto de manifiesto la necesidad de concebir el aprendizaje como un proceso de construcción activa de significados en el que las concepciones intuitivas o espontáneas de los alunnos desempeñan un papel trascendental (Driver 1981). En este contex to-la investigación acerca de la estructura cognitiva de los alumnos, actitudes hacia el aprendizaje, etc.-, ha adquirido una considerable importancia, cono muestra la amplia bibliografía existente (Gilbert y Watts 1983).
Paralelamente, se ha manifestado la necesidad de profundizar en los aspectos históricos y lógicos de la ciencia desde una perspectiva didáctica (Herron et al. 1977, Dusch 1985, Matthews 1990). Si tenemos en cuenta que la construcción de conceptos científicos aparece como núcleo central del proceso de aprendizaje (Hewson 1981, Posner et al. 1982), la reflexión y análisis de los mismos bajo el marco teórico de la las propias disciplinas cientificas, pero incorporando las aportaciones de la historia y la filosofía de la ciencia, puede ser relevante para una 
mejor comprensión de la problemática del aprendizaje.

De hecho, del mismo modo que el aprendizaje debe considerarse como un proceso dinámico, complejo e interactivo (Driver y Frikson 1983), los propios conceptos científicos deben ser juzgados en su complejidad y dinamismo. En particular, el concepto de masa, pese a su manificsta familiaridad, ofrece un claro ejemplo de la necesidad y dificultades de un análisis historico y lógico cuidadoso.

En un trabajo previo (Doménech et al., en prensa), se ha propuesto una clasificación de los diferentes conceptos de masa fundamentada en sus connotaciones propiamente físicas, semánticas y cognitivas, analizando en base a ella las definiciones dadas por una amplia muestra de estudiantes de bachillerato. Fn este trabajo se intenta ofrecer un estudio específico de las definiciones clásicas de masa desde la perspectiva de la representación física, tćrmino acuñado por Gorodetsky et al. (1986), y que se centra en la contextualización semántica de las definiciones.

\section{LOS CONCEPTOS Y LAS DEFINICIONES EN 1 AS CIENCIAS EXPERIMENTALES}

I:l problema de la definición de los conceptos utilizados en las ciencias experimentales es complejo, y puede abordarse desde diferentes puntos de vista filosóficos. En su sentido más amplio (Hempel 1988), una definición puede considerarse como una estipulación que relaciona explícitamente determinados entes entre sí, de forma que uno de ellos, el definendum, se extrae de determinadas relaciones entrc otros, el definens, cuyo significado ya está determinado.

Las dificultades surgen, sin embargo, cuando tratamos de concretar las condiciones en que se establecen las definiciones: esto es, de los requisitos lógicos y metodológicos que deben cumplir. En el fondo subyace la controversia entre las distintas escuelas de pensamiento acerca de la estructura lógica del conocimiento científico, de la naturaleza de los conceptos empleados y su conexión con el mundo físico (Bunge 1973, Naael 1978 y Hegenberg 1979). No es posible presentar aquí ni siquiera una visión panorámica de estas cuestiones: no obstante, citemos como ejemplos paradigmáticos la polémica acerca de la interpretación convencionalista de la ciencia, Ia controversia en torno a la escuela positivista o el debate sobre la interpretación probabilística de la Física Cuántica (Selleri 1986).

Aunque estos problemas pudieran parecer un tanto alejados de la praxis educativa, existen buenas razones para conceder importancia a la reflexión en torno a la estructura lógica de los conceptos y definiciones. Por un lado, por el papel desempeñado por éstas en lo que pudiésemos denominar el conocimiento estipulativo. Por otro, porque la propia estructura definicional se concreta necesariamente con esquemas de conocimiento procedural, asimismo esencial en el aprendizaje. Los problemas semánticos tampoco son ajenos: como ejemplo inmediato, la utilización đel término masa en la Física Clásica para designar entidades definicionalmente diferentes, masa inercial y masa gravitatoria.

En este sentido, es necesario tener en cuenta la complejidad del proceso de crecimiento de la ciencia, asimismo objeto de debate. Así por ejemplo, podría hablarse, bien de Ia evolución de los conceptos científicos, bien de su multiplicidad en diferentes paradigmas teóricos. Fi concepto de masa es sumamente ilustrativo al respecto, de manera que podríamos decir que el concepto de masa inercial en la relatividad especial supone una evolución del concepto new toniano de masa, diferente, a su vez, de la inercia keplcriana (Holton 1983), pero también podríamos considerarlos como conceptos independientes, insertos en contextos teóricos no comparabies, en la línea del pensamiento de Kuhn (1971). L a tabla Ipresenta las características principales de la muy específica concepción newtoniana de masa.

Tabla I

Características de la concepción clásica de masa.

\begin{abstract}
* La masa es una propiedad universal de los cuerpos: todos los objetos poseen una masa característica que sería la misma para cualquier observador

* Se trata de una magnitud escalar que se expresa por un cocficiente positivo.

* La masa se concibe como magnitud caracteristica de los sistemas materiales por oposición a espacio y tiempo que se conciken como entidades independientes de aquéllos.

* I. a masa es aditiva por acumulación, al reunir varios objetos, Ja masa del conjunto es la suma de las masas de los objetos individuales. Esta adjtividad se traduce en el principio de conservación de la masa en un sistema aislado.

* I a masa de un cuerpo es independicrte de su posición, movimiento o típo de interacción al que está sometido.

* La masa incrcial se concibe como medida de la inereia de los cuerpos tendencia a mantener su estado de reposo o movimiento rectilíneo y uniforme bajo la acción de cuajquier tipo de fuerzas.

* La masa gravitatoria se concibe como medida de la tendencia de los cuerpos a ejercer fuerzas gravitatorias entre sí: sería la mata nitud activa, responsable, de un tipo específico de interuccion, la gravitatoria.

* Masa inercial y masa gravitatoria se consideran como equivalentes.
\end{abstract}

\section{LA MASA COMO «QUANTITAS MATERIAE»}

La masa se introduce en el Libro I de los Principia en su definición primera (Newton 1982):

"La cantidad de materia es la medida de la misma, surgida de su densidad y magnitud conjuntamente.»

Esta definición supone una concepción ontológica de masa como una propiedad privilegiada y esencial de la materia. La literatura científica posterior ha retenido la 
expresión cantidad de materia, bien como una definición explícita, bien como un significado implícito de la magnitud física masa. De ahí surge una amplia tradición que considera masa y materia prácticamente como sinónimos.

Parece evidente, sin embargo, la necesidad de dotar a esta definición de un contenido empírico, de ahí que en Ios Principia la definición de masa como «quantitas materiaen va seguida, inmediatamente, de una propuesta de medida a partir del producto densidad $\times$ volumen. Esta redefinición puede parecer sorprendente desde la perspectiva actual, ya que existe una circularidad manifiesta; la densidad solamente puede ser definida, precisamente, como la relación masalvolumen. No obstante, como Brown (1960) ha señalado, la circularidad de la definición queda rota desde la perspectiva newtoniana, ya que el término densidad se utiliza en el sentido en el que actualmente se usa la llamada densidad relativa, susceptible, por otra parte, de medida directa con diferentes tipos de densímetros.

Las versiones postnewtonianas han insistido, con notable frecuencia, en dar preferencia a la vertiente metafísica de la definición como cantidad de materia. Sin embargo, la definición newtoniana claramente especifica que se trata de una medida resultado de una serie de operaciones definidas, to que nos acerca a la idea actual de magnitud física.

Esta definición de masa como medida de la cantidad de materia puede conectarse con la voluntad newtoniana de explicitar el modelocosmológico subyacente. En él, materia, espacio y tiempo aparecen como entidades separadas e independientes, dotadas de una serie de propiecades definidas (Doménech 1985).

A este respecto es obligado señalar que el modelo newtoniano nace en contraposición con la cosmología cartesiana (Cohen 1983), no solamente en cuanto a su mecánica (las fuerzas newtonianas en oposición a los vórtices cartesianos) sino en el sustrato cosmológico básico: es por ello que en los Principia incluye las propicdades de espacio y tiempo en su muy conocido escolio.

El éxito de la física newtoniana ha arrinconado por completo la cosmología cartesiana y la tajante distinsión newtoninana entre espacio, tiempo y materia forma parte de nuestro sustrato cuItural. Más aún, puesto que la dinámica newtoniana se asienta sobre los conceptos absolutos de espacio y tiempo, el concepto de materia (y por tanto, de masa) también son absolutos: la masa des un objeto será, por tanto, una propiedad universal del mismo.

Pese a todo, la trađición cosmológica cartesiana se transmite, en cierto modo, a través de las teorías de campo, lo que permite hablar de dos líneas de pensamiento: acción a distancia y acción por campos que se evolucionan paralelamente (Berkson 1981). No obstante, la difusión preferencial de la cosmología newtoniana ha determinado la permanencia de una terminología vaga que deja traslucir una serie de concepciones que, cuanto menos, podemos calificar de cuestionables. Son buen ejemplo de ello la contraposición materia-energía o masa-energía, la distinción antre materia y antimateria, materia y radiación, etc. Los enunciados de la famosa ecuación de Einstein $\mathrm{E}=\mathrm{mc}^{2}$ como interconversión materia-energía, desmaterialización y aniquilación, transformación de materia en radiación (Warren 1976, Leherman 1982, Doménech 1985, Gil et al., 1988), ampliamente extendidos, se inscriben en la mencionada concepción ontológica de masa.

\section{MASA INERCIAL, Y MASA GRAVITATORIA}

Estrictamente, masa inercial y masa gravitatoria son magnitudes diferentes, susceptibles de diferentes tipos de definición cuya eventual relación podría decidirse por vía experimental (Brown 1960). La constatación galileana de la igualdad de las aceleraciones de caída libre de los cuerpos en la superficie terrestre, las experiencias newtonianas con péndulos, así como las históricas experiencias de Eötvös y una serie de experimentos recientes (Misner et al., 1973) permiten hablar de la proporcionalidad entre masa inercial y masa gravitatoria (Einstein 1980), pero también podría hablarse en términos de equivalencia o incluso identidad. En este sentido, hay que destacar que la proporcionalidad/equivalencia entre ambos tipos de masa, aceptada como un axioma implícito en la mecánica clásica, desempeña un papel capital en la relatividad general (Sánchez-Ron 1983). En cualquier caso, el problema de la definición de masa en el ámbito relativista (especial y general) posee una serie de aspectos de mayor complejidad en los que no entraremos (Price 1982).

\section{Las definiciones funcionales}

En los Principia aparece una segunda definición explícita de masa como «vis inertiae», esto es, como medida de la inercia de los cuerpos, de su resistencia a sufrir cambios en su movimiento. Paralelamente, una segunda magnitud, que posteriormente se denominó masa gravitatoria, aparece implícitamente definida. Se trata de una propiedad semejante a la carga eléctrica, responsable, como ésta, de un determinado tipo de interacción entre los cuerpos, que mediría la «pesadez» de los mismos.

Al margen de la dicotomía entre masa inercial y masa gravitatoria, una interpretación ampliamente extendida es la que identifica masa con inercia o "pesadez», Io cual constituye lo que hemos dado en denominar una definición funcional: la magnitud así definida se conctbe como responsable de la posesión de determinadas propiedades o tendencias, o el ejercicio de determinadas respuestas u operaciones por los sistemas físicos a los que se atribuye. En esta clase definicional podria integrarse la conocida definición de energía como capacidad para producir trabajo.

De entrada, estas definiciones suponen una conexión directa con determinados observables, en función de los cuales podrían expresarse sin conexión necesaria con un 
marco térico concreto. Ésta es, sin embargo, una pretensión apresurada; así por ejemplo, la definición de masa gravitatoria como propiedad responsable de la aparición de fuerzas gravitatorias entre los cuerpos supone implícita o explíctamente el empleo del concepto de fuerza.

\section{Las definiciones relacionales y transposicionales}

Partiendo de la usual versión postnewtoniana de la segunda ley del movimiento, la masa inercial $m$, se definiría como un coeficiente de proporcionalidad, característico de cada cuerpo, obteniđo como relación entre las fuerzas sobre él aplicadas y las aceleraciones que experimenta; $F=m_{1}, a$. La masa gravitatoria podría defínirse a partir de la ley de gravitación universal o, entre otras posibilidades, de la relación fuerza gravitatoria $=$ masa gravitatoria $\times$ campo gravitatorio (Einstein 1980).

Estas definiciones pueden considerarse como relacionales, esto es, en ellas el definendum deriva del definens por medio de una relación matemática específica. Ahora podría pensarse que este tipo de definiciones es puramente convencional, $y$, en último extremo, carente de conexión empírica. Este problema se inscribe, por otra parte, en el amplio debate suscitado por la clucidación del denominado estatus lógico de las leyes clásicas del movimiento (Eisebud 1958, Nagel 1978), sobre el que existen muy diversas perspectivas.

En cualquier caso, parece existir acuerdo en cuanto a que en estas definiciones existen implicaciones de índole inequívocamente empírica. Así, la definición de masa inercial a partir de la relación $\mathrm{F} / \mathrm{a}$ supone: a) independencia respecto al tipo de fuerzas emplcadas, $b$ ) independencia de la masa con respecto a los valores de fuerzas y aceleraciones.

Las definiciones relacionales son abundantes en la Física (por ejemplo, la resistencia como constante de proporcionalidad diferencia de potencial/intensidad) y, en último término, llevarían a las que podemos denominar transposicionales. En ellas el definendum recibe su significado por identificación, oposición o condicionante directo de un definens. Así, la definición de calor específico como «el calor necesario para...». De esta forma, la masa es frecuentemente definida como peso, volumen o densidad por los alumnos.

\section{Las definiciones operacionales}

Desde una perspectiva general, las magnitudes físicas pueden considerarse como conceptos métricos (Hempel 1988), expresables como números que derivan de medidas directas o de cálculos. Esto impírica la existencia de determinadas conexiones empíricas que se concretan en mediciones y, por tanto, suponen la elección de procedimientos, instrumentos y unidades de medida.

A lo largo del primer tercio del presente siglo, adquirió una marcada difusion el enfoque operacionalista, centrado en los trabajos de Mach y Bridgman, que pone un acento especial en la dimension experimental de los conceptos fisicos (Nagel 1978). En su versión extrema, las teorías físicas podrían expresarse exclusivamente en términos de enunciados sobre observaciones empíricas y deberían incluir únicamente magnitudes cuya definición pudiera realizarse en términos de procedimientos experimentales.

Aunque la perspectiva operacionalista radical parece hoy superada, persiste, de alguna manera, un operacionalismo matizado en nuestra visión de las ciencias experimentales. De hecho, podría entenderse como una especie de regla de selección para ajustar nuestros modelos a la constrastación empírica.

Bajo un prisma operacional, la masa gravitatoria podría definirse escuetamente como «lo que mide una balanza»; en otros términos: clegido un cuerpo patrón de rererencia, la masa gravitacional de un cuerpo problema respecto al patrón podrá defínirse como la relación entre sus pesos en un punto dado de la superficie terrestro medida con una balanza de brazos iguales.

A su vez, la medida de masas inerciales podría realizarse a partir de la medida de fuerzas y accleraciones partiendo de la definición usual de masa como fuerza/aceleración. Así, dado un objeto patrón, podríamos determinar la masa de un objeto problema en relación al patrón comparando sus aceleraciones bajo la acción de una fuerza dada. Sin embargo, como ya señalara Mach en 1895 , cste procedimiento tropieza con un problema de circularidad, ya que no es posible realizar una definición de fuerza que sea completamente independiente de la de masa (Mache 1942). La idea central es que no es posible enunciar consistentemente una definición de fuerza independiente de la definición de masa y que una eventual definición operacional de fuerza (a partir de un dinamómetro convencional) es inviable (Brehme 1985).

En la formulación de Mach de la dinámica clásica, la masa inercial de un cuerpo problema se obtendría on relación a la masa de un objeto patrón como la relación inversa entre sus aceleraciones cuando se encuentran sometidos exclusivamente a su mutua interaccion, por

\section{Tabla II}

Características de las diferentes definiciones de masa.
Inscrción en un marco Relación com la teórico determinado experimentación

\begin{tabular}{lll}
\hline Ontológica & Intercambiable & Inexistente \\
Funcional & Inespecífica & Indirecta \\
Relacional & Específicá & Indirecta \\
Transposicional & Inespecífica & Indirecta \\
Operacional & Inespecífica & Directa \\
\hline
\end{tabular}


ejemplo: $\mathrm{m} / \mathrm{m} .=-\mathrm{a} / \mathrm{a}$. Por consiguiente, las masas podrían determinarse a partir de la medida de aceleraciones.

Este enfoque ha sido reiteradamente criticado en base a diferentes consideraciones de orden lógico y metodológico (Jammer 1961, Bunge 1966) que no serán analizadas aquí. Pese a ello, las definiciones operacionales podrían ser de interés didáctico en la medida en que hacen patente el carácter métrico de los conceptos físicos definidos. De hecho, es posible proponer procedimientos de medida/definiciones operacionales de masia inercial derivadas de la medida de aceleraciones (Zimmerer 1983, Goodinson and Luffman 1985) o basadas en las leyes clásicas de conservación de la cantidad de movimiento y la energía (Brehme 1985, Doménech 1985, Doménech y Doménech 1988).

\section{IMPLICACIONES DIDÁCTICAS}

Bajo una perspectiva educativa, la problemáticat que rodea al concepto o conceptos de masa podría traducirse en dilucidar qué definición o definiciones resultan didácticamente significativas y teóricamente consistentes. - Más aún, puesto que estos tipos definicionales pueden extenderse a otras magnitudes físicas sin dificultad, cabría valorar la conveniencia de adoptar determirados patrones definicionales en la enseñanza de las ciencias. Evidentemente, esta discusión pecaría de apresurada si se limitase a establecer un orden de prioridades en cuanto al tipo definicional, por cuanto la comple:idad del problema es mucho mayor, habida cuenta de sus implicaciones lógicas y metodológicas. Sin embargo, puede ser de interés comparar las características de las diferentes definiciones en cuanto a su inserción en un contexto teórico específico y su eventual conexión con operaciones empiricas (Tabla II).

Bajo esta doble perspectiva, cabe decir que la concepción ontológica de masa como cantidad de materia, pero también su versión métrica como medida de la misma, parecen rechazables en tanto en cuanto no puede adscribirse a un marco teórico específico (cabría como tâl en cualquier teoría) y carece de conexión experimental relacionada con la propia defínición. De hecho, su mantenimiento en el lenguaje cotidiano, pero también en el científico obedecería a causas complejas que reflejarl las dificultades del cambio conceptual (Hashweh 1986) entre las que se hallaría la pervivencia de una cosmovisión newtoniana simplista. El reciente debate en torno al concepto de energía (Warren 1982, Watts 1983, Duit 1987) es ilustrativo de la importancia y extensión de la misma.

Sin embargo, parece en extremo diff́cil prescindir de una terminología ampliamente extendida y a duras penas reemplazable, por lo que un objetivo razonable podría ser el de asumir críticamente el alcance estricto de la misma. En otras palabras, el problema a enfrentar no sería tanto la pervivencia de la noción de masa como cantidad de materia, como la inexistencia de concepciones más elaboradas, y la omisión de su carácter métrico.

A su vez, las definiciones funcionales, relacionales y operacionales podrían entenderse como complementarias. Las primeras son también utilizables con significado variable, pero posibilitan una formulación piausiblemente intuitiva de la dinámica $\mathrm{y}$, además, permiten una interpretación métrica directa. Por el contrario, se prestan a una visión cualitativa y teleológica de la ciencia. Las definiciones relacionales hacen claramente patente su carácter estipulativo, exigiendo una contextualización teórica explícita, pero, en el caso de la definición de masa, poseen el inconveniente de sugerir una visión privilegiada del concepto de fuerza. Por último, las definiciones operacionales podrían aportar un componente empírico necesario que reforzase el carácter métrico de las magnitudes físicas: su principal inconveniente radicaría en la posibilidad de concebirse como aisladas de un contex to teórico bien determinado.

Las anteriores consideraciones deben entenderse como base para un análisis más pormenorizado que debe tener en cuenta la tradición filosófica subyacente en los diferentes tipos definicionales. Así, las definiciones no métricas se insertarían en el ámbito de lo que Gilbert y Watts (1983) denominan tradición verstehen, cualitativa y holística; mientras que las definiciones métricas se incorporarían a la tradición erklären, cuantitativa y reduccionista. Cualquier aproximación a la didáctica de las ciencias debería tener en cuenta la complejidad del proceso de construcción de significados por el individuo, pero también la propia complejidad de los conceptos implicados: de ahí que la conexión entre los aspectos filosóficos, históricos y cognitivos (Hodson 1985, Aarons 1988) pueda considerarse como necesaria en el ámbito de la investigación didáctica.

\section{REFERENCIAS BIBLIOGRÁFICAS}

AARONS, A.B., 1988. Historical and philosophical perspectives attainable in introductory physics courses, Educarional Philosophy and Theory, Vol. 19, pp. 425-432.

BERKSON, W., 1981. Las teorías de los campos de fuerza. Desde Faraday hasta Einstein. (Alianza Editorial: Madrid).
BREHME, R.W., 1985. On force and the inertial frame, American Journal of Physics, Vol. 53, pp. 952-955.

BROWN, G.B., 1960. Gravitational and Inertiai Mass, American Journal of Physics, VoI. 28, pp. 475-483. 
BUNGE, M. 1966. Mach's Critique on Newtonian Mechanics, American Journal of Physics, Vol. 34, pp. 585-596.

BUNGF, M., 1973. La investigación cientifica. (Ariei: Barceiona).

COHFN, L.B., 1983. La revolución newtoniana y la transformacion de las ideas científicas. (Alianza Editorial: Madrid).

DOMÉNECH, A., 1985. Aproximación al estudio de espacio y tiempo. (ICE Universidad de Valencia: Valencia).

DOMÉNECH, A. y DOMÉNECH, M.T., 1988. Relationships hetween the scattering angles in pendulum collisions, European Journal of Physics, Vol. 9, pp. 117-122.

IOMÉNECH, A., CASASÚS, E. y DOMÉNECH, M.T. (en prensa). The classical concept or mass. Theoretical difficulties and students definitions, International Journal of Science Education.

DRIVER, R., 1981. Pupil's alternative frameworks in science, European Journal or Science Education, Vol. 10, pp. 37-60.

DRIVER, R, y ERICKSON. G., 1983. Theories-in-action: Some theoretical and empirical issues in the study of students' conceptual frameworks in science, Studies in Science Education, Vol. 10, pp. 37-60.

DUIT, R., 1987. Should energy be illustrated as something quasi-material?, International Journal of Science Educa. tion, Vol. 9, pp. 139-145.

DUSCH, R.A., 1985. Science Education and Philosophy of Science, twenty-five years or mutually exclusive development, School Science and Mathematics, Vol. 87, pp. 541555 .

EINSTEIN, A., 1980. El significado de la relatividad. $4^{\mathrm{A}} \mathrm{ed}$. (Espasa-Calpe: Madrid).

EISF:BUD, I,., 1958. On the Classical laws of motion, American Journal of Physics, Vol. 26, pp. 144-159.

GIL, D., SENENT, F. y SOLBES, J., I988. E=mc ${ }^{2}$, la ecuación más famosa de la física: Una incomprendida, Revista Española de Física, Vol. 2, pp. 53-55.

GILBERT,J.K. y WATTS, D.M. 1983. Concepts, Misconceptions and Alternative Conceptions: Changing Perspectives in Science Education, Studies in Science Education, Vol. 10, pp. 61-98.

GOODINSON, P.A., y LUFFMAN, B.L., 1985. On the definition of mass in classical physics, American Journal of Physics, Vol. 53, pp. $40-42$

GORODFTSKY, M., HOZ, R. y VINNER, S., 1986. Hierarchical Solution Models of Speed Problems, Science Education, Vol. 70, pp. 565-582.

HASHWEH, M.Z., 1986. Towards an explanation of conceptual change, European Journal of Science Education, Vol. 8, pp. 229-249.

HEGENBERG, L., 1979. Introducción a la filosofía de la ciencia $2^{3}$ ed. (Herder: Barcelona).

HFMPEL, C.G., 1988. Fundamentos de la formación de conceptos en ciencia empirica. (Alianza Fditorial: Madrid).
HERRON, J.D., CANTU, U., WARD, R. y SRINIVANSON, V., 1977. Problems associated with concept analysis, Science Education, Vol. 61, pp. 185-199.

HEWSON, P.W., 1981. A conceptual change approach to leaming science, European Journal of Science Education, Vol. 3, pp. 383-396.

HODSON, D., 1985. Philosophy of Science. Science and science Fducation, Studies in Science Education, Vol. 12, pp. $25-27$

HOLTON, G., 1983. Introducción a los conceptos y teorias de las ciencias físicas, $2^{\mathrm{i}}$ ed. (Reverté: Barcelona).

JAMMER, M., 1961. The Concepts of Mass. (Harvard Univ. Cambridge: Mass.).

KUHN, 'T, S., 1971. La estructura de las revoluciones cientificas. (Fondo de Cultura Económica: Madrid).

I.EHERMAN, R, I., 1982. Confused Physics: A tutorial critique. The Physics Teacher, Vol. 20, pp. 519-523.

MACH, E., 1942. The Science of Mechanics. (Open Court Pub. La Salle).

MATTHEWS, M.R., 1990. History, Philosophy and Science Teaching: a rapprochement, Studies in Science Education, Vol. 18, pp. 25-51.

MISNER, C.W., THORNE, K.S. y WHEELER. J.A., 1973. Gravitation. (Freeman \& Co.: San Francisco).

NFWTON, I., 1982. Principios Matemáticos de la Filosofia Natural y su Sistema del Mundo. (Fditora Nacional. Madrid).

NAGEL, E., 1978. La Estructura de la ciencia. (Paidós: Buenos Aires).

POSNER, G., STRIKE, K., HEWSON. D. y GERTZOG. W. 1982. Accomodation of a scientific conception: toward a theory of conceptual change. Science Education, Vol. 66 pp. 211-227.

PRICE, R.H., 1982. General relativity primer, American Journal or Physics, Vol. 50, pp. 300-329.

SÁNCHEZ-RON., J.M., 1983. El origen y desarrollo de la relatividad. (Alianza Editorial: Madrid).

SELLERI, F., 1986. El debate de la teoria cuántica. (Alianza Editorial. Madrid).

WARREN, J.W., 1976. The mystery or mass-energy, Physirs Education, Vol. 11, pp. 52-54.

WARREN, J.W., 1982. The nature of energy, European Journal of Science Education, Vol. 4, pp. 295-297.

WATTS, M.D., 1983. Some alternative views of energy, Physics Education, Vol. 18, pp. 213-217.

ZIMMERER. R.W., 1983. Meastrement of mass, Physics Teacher, Vol. 21, pp. 354-359, 